\title{
HARLOW SHAPLEY AND RED GIANT STARS
}

Martin F. McCarthy S.J.

Vatican Observatory

In five papers written between 1951 and 1955 Shapley considers the topic of red giant stars and reddish variable stars in the Magellanic Clouds. These works coincide with Shapley's final year as Director at Harvard and the first years of his retirement which extended a full score of years before his death in 1972. They include the following: Magellanic Clouds II (Supergiants/Red Variable Stars in the Small Cloud; January 1951); Magellanic Clouds IV (On Period Frequency Anomalies; February 1952); Magellanic Clouds VII (Star Colors and Luminosities in Five Constellations; March 1953); Magellanic Clouds VIII (On the Populations Characteristics of the Two Clouds; October 1953); and Magellanic Clouds XVI (Infrared Stars and Stellar Evolution; July 1955). These five papers, which appeared originally in the Proceedings of the National Academy of Science, may be found in the Harvard Reprint Series $I$ as numbers $346,360,373,376$, and 425 .

Here Shapley explores the brightness and the size of the stars in the Clouds, then realizes that the domain of the main sequence and much of the giant branches must remain projects for future studies and limits himself to a first survey and examination of the stars of high luminos-

ity. Bounds were imposed on Shapley's research on giants in the Clouds because he did not have available sufficient material for spectral classification and for radial velocity measures of the faint stars. Lacking these he could not with certainty separate Cloud members from foreground stars. He used what he had: preliminary magnitudes and colors plus measures of the variations of the red variables, where he built upon the measures of C.P. and S. Gaposchkin as well as the photometric measures of Nail; he was aware of the importance of interpolating magnitudes on photographic plates from reliable photoelectric photometry and he used the new sequences developed by Uco van Wyck. Shapley obtained the best available distance moduli to the Clouds and argued from a comparison of star fields outside the Clouds at comparably high galactic latitudes to a discrimination between Cloud members and superposed stars of the galaxy. He estimated absorption effects as carefully as possible but realizes he has no substitute for actual colors derived from normal colors based on spectral studies.

While these studies are overshadowed by his earlier and greater works on the galactic center, Shapley stands for us as the Gatekeeper to the realms of the Magellanic Clouds. He yearned to explore these in 
detail but was limited in his access and knew he could not enter these Promised Lands fully. When he came to the end, he found that it was only the beginning. Today those who understand so much more about the size, structure and development of objects in the Clouds than Harlow Shapley could thank him for affording to them something of his vision and his giant's shoulders.

The text of this paper will be published fully elsewhere. 